\title{
$L^{2}$-DOLBEAULT COMPLEXES ON SINGULAR CURVES AND SURFACES
}

\author{
PETER HASKELL
}

(Communicated by Frederick R. Cohen)

\begin{abstract}
Give the smooth part of a singular curve or normal surface the metric induced from the ambient projective space. On this incomplete manifold the minimal $L^{2} \bar{\partial}$-complex of $(0, q)$-forms has finite-dimensional cohomology groups. The Euler characteristic of this cohomology equals the Todd genus of any desingularization of the singular variety.
\end{abstract}

R. MacPherson ([4], [8]) has conjectured that the smooth part of a singular algebraic variety (when given one of certain natural metrics) carries an $L^{2} \bar{\partial}$ complex of $(0, q)$-forms whose Euler characteristic is equal to the Todd genus of any desingularization of the variety. Much progress on this conjecture has been made using complete metrics on the smooth parts of the varieties. The present paper focuses on the incomplete metrics induced from the ambient projective spaces. W. Pardon [10] has written an important paper showing that when $X$ is a curve or normal surface whose smooth part is given the metric induced from the ambient projective space, then the complex of smooth $(0, q)$-forms $w$ satisfying $w \in L^{2}$ and $\bar{\partial} w \in L^{2}$ has an Euler characteristic that is not necessarily equal to the Todd genus of a desingularization of $X$. However, an earlier paper [6] exhibited a $\bar{\partial}$-complex on the smooth part of a curve $X$ (with metric induced from the ambient projective space) that has the Euler characteristic conjectured by MacPherson. In the search for the complex whose existence is conjectured by MacPherson, one must choose carefully a closed extension of the $\bar{\partial}$-operator.

Let $X$ be a complex projective curve or normal surface, and let $U$ be the set of smooth points of $X$. Give $U$ the metric induced from the ambient projective space. The present paper shows that the minimal $\bar{\partial}$-complex (defined in Definition 1.5) of $(0, q)$-forms on $U$ has finite-dimensional cohomology groups and that these groups are isomorphic to the corresponding $\bar{\partial}$-cohomology groups on any desingularization of $X$. Thus the Euler characteristic of these

Received by the editors April 9, 1988, and in revised form December 2, 1988.

1980 Mathematics Subject Classification (1985 Revision). Primary 58G10, 58G05; Secondary 57R20, 32J25.

This material is based upon work supported by the National Science Foundation under Grant No. DMS-8717186. 
cohomology groups on $U$ equals the Todd genus of any desingularization of $X$, i.e. MacPherson's conjecture is true for these minimal complexes on curves and normal surfaces. These results suggest a rephrasing of MacPherson's conjecture in general: the Euler characteristic of the minimal $L^{2} \bar{\partial}$-complex on the smooth part of a singular variety (with induced metric) equals the Todd genus of any desingularization of the variety.

The reasoning in the present paper relies heavily on that in [10]. I thank Bill Pardon for sending me his preprint and discussing his work with me. The present paper is a revision of a previous preprint with the same title. In the original version, it was not clear that the $\bar{\partial}$-operator defined on functions on surfaces was minimal. After circulation of the original preprint, Bill Pardon and I realized independently that all the $\bar{\partial}$-operators used here are minimal.

\section{Definitions AND PRINCIPLeS}

Henceforth the smooth parts of singular varieties are given the metrics induced from the ambient projective spaces. Let $X$ be a curve or normal surface, and let $U$ be the set of smooth points of $X$. Let $\pi: \widetilde{X} \rightarrow X$ denote a desingularization of $X$. Define $E$ to be $\widetilde{X}-\pi^{-1}(U)$. Assume that $E$ has (at worst) normal crossings. In this section we describe the complexes that we use to study the minimal $\bar{\partial}$-complexes on $U$. We also state two principles, which serve to compare analysis on $U$ to analysis in a nondegenerate metric on $\widetilde{X}$ by tying each to analysis on a dense open subset of $\widetilde{X}$.

Definition 1.1. A differential form on (an open subset) of $\tilde{X}$ is called $L^{2}$ if it is $L^{2}$ in any nondegenerate Kähler metric on $\widetilde{X}$. The pullback to $\widetilde{X}$ of the metric on $U, \pi^{*}$ (metric on $U$ ), is a pseudometric on $\widetilde{X}$. We denote this pseudometric by $\gamma$. The degeneracy locus of $\gamma$ is contained in $E$. A differential form is called $L_{\gamma}^{2}$ if it is $L^{2}$ in the pseudometric $\gamma$. Except where $\gamma$ is explicitly mentioned, one should assume that we are using a nondegenerate Kähler metric on $\tilde{X}$.

A singular curve has a unique desingularization. If $X$ is a normal surface, the desingularizations we discuss are always of the following form. $E$ is a codimension one subvariety with (at worst) simple normal crossings. For each smooth point of $E$ there are integers $m$ and $n, n \geq m \geq 1$, such that locally around the point on $E, \pi^{-1}(\operatorname{sing}(X))=\left(u^{m}\right)$ as a subvariety and $\gamma$ is quasiisometric to $d \varphi d \bar{\varphi}+d \psi d \bar{\psi}$. Here $\varphi(u, v)=u^{m}$ and $\psi(u, v)=u^{n} v$. For each normal crossing of $E$ there are integers $m_{1}, n_{1}, m_{2}, n_{2}$ satisfying $n_{1} \geq$ $m_{1} \geq 1, n_{2} \geq m_{2} \geq 1$ and $m_{1} n_{2}-m_{2} n_{1} \neq 0$. Locally around the crossing point on $E, \pi^{-1}(\operatorname{sing}(X))=\left(u^{m_{1}} v^{m_{2}}\right)$ as a subvariety and $\gamma$ is quasi-isometric to $d \varphi d \bar{\varphi}+d \psi d \bar{\psi}$. Here $\varphi(u, v)=u^{m_{1}} v^{m_{2}}$ and $\psi(u, v)=u^{n_{1}} v^{n_{2}}$. In either case one can calculate that the volume form associated to the metric $d \varphi d \bar{\varphi}+d \psi d \bar{\psi}$ is locally $\left|\varphi_{u} \psi_{v}-\psi_{u} \varphi_{v}\right|^{2}$ times the standard volume form on $\mathbb{C}^{2}$. Normal 
singular surfaces always have desingularizations with these properties [7]. (My discussion uses the notation and organization of [10].)

Remark 1.2. The results of this paper are of the form: certain $\bar{\partial}$-cohomology groups on $U$ are isomorphic to the $(0, q) \bar{\partial}$-cohomology groups of $\widetilde{X}$. The proofs given apply directly to $\widetilde{X}$ of the type described above. However, because the $(0, q) \bar{\partial}$-cohomology groups are birational invariants of smooth varieties [5], the results of this paper apply to any desingularization $\pi: \widetilde{X} \rightarrow X$.

Definition 1.3. $A^{p, q}(U)=\left\{\right.$ smooth $L^{2}$ forms $w$ of type $(p, q)$ on $U$ such that $\bar{\partial} w$ is also $\left.L^{2}\right\}$. (For a form on $U, L^{2}$ means with respect to the metric induced on $U \subset X$ from the ambient projective space.) The maximal Dolbeault complex on $U$ is $\left\{A^{0, *}(U), \bar{\partial}\right\}$. The cohomology groups of this complex are $H^{0, *}(U)$. There are also complexes $\left\{A^{p, *}(U), \bar{\partial}\right\}$ with cohomology groups $H^{p, *}(U)$.

Definition 1.4. Let $\mathscr{D}_{c}^{p, q}$ denote the $\bar{\partial}$-operator defined on smooth compactly supported forms of type $(p, q)$ on $U$. Viewing $\mathscr{D}_{c}^{p, q}$ as an unbounded operator from $L^{2}$ forms of type $(p, q)$ on $U$ to $L^{2}$ forms of type $(p, q+1)$ on $U$, let $\mathrm{D}_{c}^{p, q}$ be the closure of $\mathscr{D}_{c}^{p, q}$. When the values of $p$ and $q$ are clear from context, we omit them from the notation.

Definition 1.5. Let $A_{c}^{p, q}(U)$ be the collection of forms smooth on $U$ and in the domain of $\mathrm{D}_{c}^{p, q}$. The minimal Dolbeault complex on $U$ is $\left\{A_{c}^{0, *}(U), D_{c}\right\}$, and its cohomology groups are denoted $H_{c}^{0, *}(U)$.

Definition 1.6. Let $L^{p, q}(\widetilde{X})$ denote the set of $L^{2}$ forms of type $(p, q)$ on $\tilde{X}$. Under restriction $L^{p, q}(\widetilde{X})$ is isomorphic to the set of $L^{2}$ forms of type $(p, q)$ on $\tilde{X}-E$. Let $L_{\gamma}^{p, q}(\widetilde{X})$ denote the set of $L_{\gamma}^{2}$ forms of type $(p, q)$ on $\tilde{X}-E$.

Definition 1.7. $A_{E}^{p, q}(\widetilde{X})=$ smooth $L^{2}$ forms $w$ of type $(p, q)$ on $\tilde{X}-E$ such that $\bar{\partial} w$ is also $L^{2}$ on $\left.\tilde{X}-E\right\}$. Here $\bar{\partial}$ is the $\bar{\partial}$-operator on the complex manifold $\tilde{X}-E$.

Definition 1.8. $A_{\gamma}^{p, q}(\widetilde{X})=\left\{\right.$ smooth $L_{\gamma}^{2}$ forms $w$ of type $(p, q)$ on $\tilde{X}-E$ such that $\bar{\partial} w$ is also $L_{\gamma}^{2}$ on $\left.\tilde{X}-E\right\}$. Here $\bar{\partial}$ is again the $\bar{\partial}$-operator on $\widetilde{X}-E$. The cohomology of the complex $\left\{A_{\gamma}^{p, *}(\widetilde{X}), \bar{\partial}\right\}$ is denoted $H_{\gamma}^{p, q}(\widetilde{X})$. The closures of these $\bar{\partial}$-operators are the maximal $\bar{\partial}$-operators, $\bar{\partial}: L_{\gamma}^{p, q}(\widetilde{X}) \rightarrow L_{\gamma}^{p, q+1}(\widetilde{X})$.

Definition 1.9. Let $\mathscr{D}_{c, \gamma}^{p, q}$ denote the $\bar{\partial}$-operator defined on smooth forms of type $(p, q)$ with compact support on $\tilde{X}-E$. Viewing $\mathscr{D}_{c, \gamma}^{p, q}$ as an unbounded operator from $L_{\gamma}^{2}$ forms of type $(p, q)$ on $\tilde{X}-E$ to $L_{\gamma}^{2}$ forms of type $(p, q+1)$ on $\tilde{X}-E$, let $\bar{\partial}_{c}^{p, q}$ be the closure of $\mathscr{D}_{c, \gamma}^{p, q}$. When the values of $p$ and $q$ are clear from context, we write $\bar{\partial}_{c}$ for $\bar{\partial}_{c}^{p, q}$. 
Definition 1.10. $A_{c}^{p, q}(\tilde{X})=\left\{\right.$ smooth forms on $\tilde{X}-E$ in the domain of $\left.\bar{\partial}_{c}\right\}$. The cohomology of the complex $\left\{A_{c}^{p, *}(\widetilde{X}), \bar{\partial}_{c}\right\}$ is $H_{c}^{p, q}(\widetilde{X})$.

In order to compare analysis on $U$ with analysis on $\tilde{X}$, we use the isomorphism between complex manifolds $U$ and $\widetilde{X}-E$ realized by $\pi$ to identify $U$ with $\tilde{X}-E$. This leads to the following principle.

Pseudometric principle. The following diagrams commute:

$$
\begin{array}{cccc}
A_{\gamma}^{p, q}(\tilde{X}) & \stackrel{\bar{\partial}}{\rightarrow} & A_{\gamma}^{p, q+1}(\tilde{X}) \\
\| l & & \| l \\
A^{p, q}(U) & \stackrel{\bar{\partial}}{\rightarrow} & A^{p, q+1}(U) \\
A_{c}^{p, q}(\tilde{X}) & \stackrel{\bar{\partial}_{c}}{\rightarrow} & A_{c}^{p, q+1}(\tilde{X}) \\
\| l & & \|^{2} \\
A_{c}^{p, q}(U) & \stackrel{D_{c}}{\rightarrow} & A_{c}^{p, q+1}(U)
\end{array}
$$

Thus $H^{p, q}(U) \cong H_{\gamma}^{p, q}(\tilde{X})$ and $H_{c}^{p, q}(U) \cong H_{c}^{p, q}(\tilde{X})$. There are analogous diagrams of Hilbert spaces and closed unbounded operators.

Henceforth our analysis of $\bar{\partial}$-complexes on $U$ will all be expressed in terms of analysis of the complexes on $\widetilde{X}-E$ appearing in the above principle.

Definition 1.13. $A^{p, q}(\widetilde{X})=\{$ smooth forms of type $(p, q)$ on $\widetilde{X}\}$. The complex $\left\{A^{p, *}(\widetilde{X}), \bar{\partial}\right\}$ extends to a complex of closed unbounded operators on Hilbert spaces $\left\{L^{p, q}(\widetilde{X}), \bar{\partial}\right\}$. The latter $\bar{\partial}$ can be interpreted either as the closure of the former $\bar{\partial}$ or as $\bar{\partial}$ taken in the distribution sense.

Proposition 1.14 [5]. For each $p$ the cohomology of either $\left\{A^{p, *}(\tilde{X}), \bar{\partial}\right\}$ or $\left\{L^{p, *}(\widetilde{X}), \bar{\partial}\right\}$ is the well-known Dolbeault cohomology $H^{p, q}(\tilde{X})$.

Remark 1.15. $\sum_{q}(-1)^{q} \operatorname{dim}_{\mathrm{C}} H^{0, q}(\tilde{X})$ equals the Todd genus of $\tilde{X}$.

The pseudometric principle leaves us with differential forms that do not necessarily extend smoothly to all of $\widetilde{X}$. The following principle begins to address this complication.

Proposition 1.16 [10] (Absence of residues principle). Let $\tilde{X}$ be a curve or let $\widetilde{X}$ be a surface and $E$ a divisor with (at worst) simple normal crossings. Let $r$ be restriction to $\widetilde{X}-E$ and let $i$ be inclusion. Then for each $p$ the maps

$$
A^{p, q}(\tilde{X}) \stackrel{r}{\rightarrow} A_{E}^{p, q}(\tilde{X}) \stackrel{i}{\rightarrow} L^{p, q}(\tilde{X})
$$

induce maps of complexes

$$
\left\{A^{p, *}(\tilde{X}), \bar{\partial}\right\} \rightarrow\left\{A_{E}^{p, *}(\tilde{X}), \bar{\partial}\right\} \rightarrow\left\{L^{p, q}(\tilde{X}), \bar{\partial} \text { (in the distribution sense) }\right\} .
$$

Each complex has cohomology equal to $H^{p, *}(\tilde{X})$. 
Proof. The issues addressed by this proposition center around $\left\{A_{E}^{p, *}(\tilde{X}), \bar{\partial}\right\}$. A smooth $L^{2}$ form $w$ on $\widetilde{X}-E$ is also $L^{2}$ on $\widetilde{X}$. Let $w^{\prime}$ be the image of $w$ under the $\bar{\partial}$-operator, defined in the distribution sense on $\widetilde{X}$. The restriction of $w^{\prime}$ to $\tilde{X}-E$ will agree with $\bar{\partial} w$ calculated locally and formally on $\widetilde{X}-E$. However, a priori the difference between $w^{\prime}$ and the restriction of $w^{\prime}$ to $\widetilde{X}-E$ may be nonzero, i.e. there may be a nonzero residue supported on $E$. The absence of such residues is proven for $p=0$ in [10]. The proof extends to other $p$ because this proposition involves only a nondegenerate metric on $\widetilde{X}$.

The calculation of the cohomology of the first and third complexes proceeds by showing that these complexes are composed of global sections of fine resolutions of appropriate sheaves. (See e.g. [5].) Pardon [10] calculates that the same reasoning applies to the middle complex.

Proposition 1.17. Let $n=\operatorname{dim}_{\mathrm{C}} X$. Then there are inclusions of complexes

$$
\left\{L_{\gamma}^{n, *}(\tilde{X}), \bar{\partial}\right\} \subset\left\{L^{n, *}(\tilde{X}), \bar{\partial}\right\}
$$

and

$$
\left\{A_{\gamma}^{n, *}(\tilde{X}), \bar{\partial}\right\} \subset\left\{A_{E}^{n, *}(\tilde{X}), \bar{\partial}\right\} .
$$

There are also inclusions of complexes

$$
\left\{L^{0, *}(\tilde{X}), \bar{\partial}\right\} \subset\left\{L_{\gamma}^{0, *}(\tilde{X}), \bar{\partial}_{c}\right\}
$$

and

$$
\left\{A_{E}^{0, *}(\tilde{X}), \bar{\partial}\right\} \subset\left\{A_{c}^{0, *}(\widetilde{X}), \bar{\partial}_{c}\right\} .
$$

Proof. The first two inclusions follow directly from a comparison of the norms assigned to forms of type $(n, *)$ by a nondegenerate metric on $\widetilde{X}$ and by the pseudometric $\gamma$. The third and fourth inclusions would have a similar proof if $\bar{\partial}_{c}$ were replaced by the maximal $\bar{\partial}$ and $A_{c}^{0, *}(\widetilde{X})$ by $A_{\gamma}^{0, *}(\widetilde{X})$. The difficulty is that $\bar{\partial}_{c}$ is the closure of an operator defined on forms with compact support in $\tilde{X}-E$. It is not obvious, a priori, that a $(0, *)$-form on $\widetilde{X}-E$ that extends to a smooth form on $\widetilde{X}$ is in $A_{c}^{0, *}(\widetilde{X})$. However, in the inclusion of complexes in Proposition 1.16 (for $p=0$ ), the right-hand (distribution) $\bar{\partial}$ is the closure of the left-hand one (on $A^{0, *}(\widetilde{X})$ ), when we view both as unbounded operators on $L^{0, *}(\tilde{X})$. It follows that to establish our inclusion it suffices to show that any form in $A^{0, *}(\widetilde{X})$ can be approximated in the graph norm of the $\bar{\partial}$-operator by forms compactly supported on $\widetilde{X}-E$. Because the restriction of a nondegenerate metric on $\widetilde{X}$ to $\widetilde{X}-E$ is conical, this sufficient condition is satisfied: the restriction of an element of $A^{0, *}(\widetilde{X})$ to $\widetilde{X}-E$ is in the domain of maximal exterior differentiation, which equals the domain of minimal exterior differentiation [2]; the estimates that are equivalent to this equality of domains are sufficient to show that the restriction of an element of $A^{0, *}(\widetilde{X})$ to $\widetilde{X}-E$ is in the domain of the minimal $\bar{\partial}$-operator. 


\section{NORMAL SURFACES}

In this section $X$ is a normal surface with smooth part $U . U$ has the metric induced from the ambient projective space. This section is devoted to proving that the cohomology groups of the minimal $\bar{\partial}$-complex of $(0, *)$ forms on $U$ are isomorphic to the corresponding groups $H^{0, *}(\widetilde{X})$ on a special (and thus any) desingularization of $X$. The minimal $\bar{\partial}$-complex on $U$ is the complex $\left\{A_{c}^{0, *}(U), D_{c}\right\}$. The pseudometric principle states that this complex is isomorphic to $\left\{A_{c}^{0, *}(\tilde{X}), \bar{\partial}_{c}\right\}$. We work with the latter complex.

Theorem 2.1. Let $X$ be a normal surface and let $\pi: \widetilde{X} \rightarrow X$ be a desingularization of the type described just before Remark 1.2. Then the inclusion of complexes

$$
\left\{A_{E}^{0, *}(\tilde{X}), \bar{\partial}\right\} \subset\left\{A_{c}^{0, *}(\tilde{X}), \bar{\partial}_{c}\right\}
$$

established in Proposition 1.17 induces an isomorphism of cohomology groups

$$
H^{0, q}(\tilde{X}) \cong H_{c}^{0, q}(\tilde{X}) \text {. }
$$

Proof. The proof of this theorem consists of the proofs of three propositions.

Proposition 2.2. $\left\{A_{E}^{0, *}(\widetilde{X}), \bar{\partial}\right\} \rightarrow\left\{A_{c}^{0, *}(\widetilde{X}), \bar{\partial}_{c}\right\}$ induces $H^{0,0}(\widetilde{X}) \cong H_{c}^{0,0}(\widetilde{X})$.

Proof. Constant functions are in the domain of minimal exterior differentiation on $\widetilde{X}-E$ [9], and thus they are in domain $\left(\bar{\partial}_{c}\right)$. Nothing else is in kernel $\left(\bar{\partial}_{c}\right)$. [10]

Proposition 2.3. $\left\{A_{E}^{0, *}(\tilde{X}), \bar{\partial}\right\} \rightarrow\left\{A_{c}^{0, *}(\tilde{X}), \bar{\partial}_{c}\right\}$ induces $H^{0,2}(\tilde{X}) \cong H_{c}^{0,2}(\tilde{X})$.

Proof. An explicit calculation with the Hodge *-operator shows that $L^{0,2}(\widetilde{X})$ is isomorphic to $L_{\gamma}^{0,2}(\widetilde{X})$. By Proposition 1.17 we have a commutative diagram:

$$
\begin{array}{ccc}
L^{0,1}(\tilde{X}) & \stackrel{\bar{\partial}}{\rightarrow} & L^{0,2}(\widetilde{X}) \\
\mathfrak{L} & & \| 2 \\
L_{\gamma}^{0,1}(\widetilde{X}) & \stackrel{\bar{\partial}_{\mathcal{c}}}{\rightarrow} & L_{\gamma}^{0,2}(\widetilde{X}) .
\end{array}
$$

Thus $H^{0,2}(\widetilde{X})$ maps surjectively to cokernel $\left(\bar{\partial}_{c}^{0,1}\right)$, and this cokernel is finitedimensional. Range $\left(\bar{\partial}_{c}^{0,1}\right)$ is closed. We can identify cokernel $\left(\bar{\partial}_{c}^{0,1}\right)$ with $\operatorname{kernel}\left(\left(\bar{\partial}_{c}^{0,1}\right)^{*}\right)$ and thus with $\operatorname{kernel}\left(\bar{\partial}: L_{\gamma}^{2,0}(\tilde{X}) \rightarrow L_{\gamma}^{2,1}(\tilde{X})\right)$ [3]. Explicit calculation with the Hodge *-operator shows that $L_{\gamma}^{2,0}(\widetilde{X})$ is isomorphic to $L^{2,0}(\tilde{X})$. An application of Proposition 1.16 then implies that kernel $\left(\bar{\partial}: L_{\gamma}^{2,0}(\tilde{X}) \rightarrow L_{\gamma}^{2,1}(\tilde{X})\right)$ is isomorphic to $H^{2,0}(\tilde{X})$, and so cokernel $\left(\bar{\partial}_{c}^{0,1}\right)$ is isomorphic to $H^{0,2}(\widetilde{X})$.

Our arguments have also identified cokernel $\left(\bar{\partial}_{c}^{0,1}\right)$ with (part of) the kernel of an elliptic operator. The Hodge *-operator takes smooth forms to smooth forms. Thus the cokernel of $\bar{\partial}_{c}: L_{\gamma}^{0,1}(\widetilde{X}) \rightarrow L_{\gamma}^{0,2}(\widetilde{X})$ is contained in the cokernel of $\bar{\partial}_{c}: A_{c}^{0,1}(\widetilde{X}) \rightarrow A_{c}^{0,2}(\widetilde{X})$. When a preimage under $\bar{\partial}_{c}^{0,1}$ of a form 
smooth on $\tilde{X}-E$ exists, a preimage smooth on $\tilde{X}-E$ can be chosen [10]. It follows that the cokernel of $\bar{\partial}_{c}: A_{c}^{0,1}(\widetilde{X}) \rightarrow A_{c}^{0,2}(\widetilde{X})$ is isomorphic to the cokernel of $\bar{\partial}_{c}: L_{\gamma}^{0,1}(\tilde{X}) \rightarrow L_{\gamma}^{0,2}(\tilde{X})$ and thus to $H^{0,2}(\widetilde{X})$.

To prove Proposition 2.6, which states that $H^{0,1}(\tilde{X})$ is isomorphic to $H_{c}^{0,1}(\widetilde{X})$, we need two lemmas.

Lemma 2.4. The range of $\bar{\partial}_{c}: L_{\gamma}^{0,0}(\widetilde{X}) \rightarrow L_{\gamma}^{0,1}(\widetilde{X})$ is closed.

Proof. This proof is based on an argument of [10]. However, to get this result for the minimal $\bar{\partial}$-operator, we need an application of the open mapping theorem, which is unnecessary for the maximal $\bar{\partial}$-operator of [10].

The $\bar{\partial}$-complex of $L^{2}(0, *)$-forms with coefficients in a holomorphic line bundle on $\widetilde{X}$ has finite-dimensional cohomology groups. (The complex is the global sections of a fine resolution of the sheaf of holomorphic sections of the line bundle.) Let $Z_{\gamma}$ be the degeneracy divisor on $\widetilde{X}$ for the pseudometric $\gamma . Z_{\gamma}$ is an effective divisor, and as point sets the support of $Z_{\gamma}$ and $E$ are equal. Let $\mathscr{L}_{\gamma}$ be the line bundle associated to $Z_{\gamma} . Z_{\gamma}=$ $\sum\left(m_{i}+n_{i}-1\right) Z_{i}$, where the $Z_{i}$ are irreducible components. The $m_{i}$ and $n_{i}$ arise as follows. (Recall the discussion preceding Remark 1.2.) Around a smooth point of $Z$ on $Z_{i}, \gamma=d \varphi d \bar{\varphi}+d \psi d \bar{\psi}$ where $\varphi(u, v)=u^{m_{i}}$ and $\psi(u, v)=u^{n_{i}} v$. These values of $m_{i}$ and $n_{i}$ are independent of the choice of smooth point on $Z_{i}$ [10].

The $L^{2}(0, *)$-forms with coefficients in $\mathscr{L}_{\gamma}$ can be identified with $(0, *)$ forms on $\tilde{X}-E$ which are $L^{2}$ away from $Z_{\gamma}$ and whose products with the appropriate local defining functions for $Z_{\gamma}$ are $L_{\text {loc }}^{2}$ in neighborhoods of points in $Z_{\gamma}$. We give the name $\mathscr{L}^{0, *}(\tilde{X})$ to these spaces of $L^{2}$ forms with coefficients in $\mathscr{L}_{\gamma}$. The unbounded maximal operator $\bar{\partial}_{\mathscr{L}}: \mathscr{L}^{0,0}(\tilde{X}) \rightarrow \mathscr{L}^{0,1}(\tilde{X})$ is the operator referred to in the first sentence of the preceding paragraph and thus has closed range. The operator $\bar{\partial}_{\mathscr{L}}$ equals the closure of its restriction to smooth forms compactly supported in $\widetilde{X}-E$. (Because we are working in a nondegenerate metric on $\widetilde{X}$, the estimates arising in the proof of Proposition 1.17 apply here also.)

The products of $\mathscr{L}_{\gamma}^{2}$ forms with the local defining equations of $Z_{\gamma}$ are also $L_{\text {loc }}^{2}$ in neighborhoods of points in $Z_{\gamma}$. It follows that there is a commutative diagram:

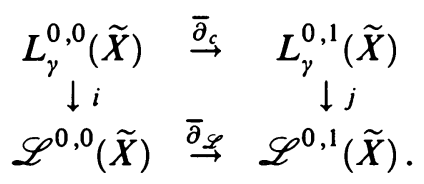

Here $i$ and $j$ are the inclusions arising from the description of $\mathscr{L}^{0, *}(\widetilde{X})$ in the preceding paragraph. 
We can now prove that $\bar{\partial}_{c}^{0,0}$ has closed range. Let $\left\{x_{n}\right\}$ be a sequence of elements of range $\left(\bar{\partial}_{c}^{0,0}\right)$ that converges to $x \in L_{\gamma}^{0,1}(\tilde{X})$. By approximating if necessary we may assume that each $x_{n}$ is the image of a smooth function $F_{n}$ compactly supported in $\tilde{X}-E$. The sequence $\left\{j\left(x_{n}\right)\right\}$ converges to $j(x)$ in $\mathscr{L}^{0,1}(\widetilde{X})$. Because $\bar{\partial}_{\mathscr{L}}$ has closed range, $j(x) \in \operatorname{range}\left(\bar{\partial}_{\mathscr{L}}\right)$. Let $f_{n}$ and $f$ be the elements of $\mathscr{L}^{0,0}(\widetilde{X})$ in the orthogonal complement of kernel $\left(\bar{\partial}_{\mathscr{L}}\right)$ and satisfying $\bar{\partial}_{\mathscr{L}}\left(f_{n}\right)=j\left(x_{n}\right)$ and $\bar{\partial}_{\mathscr{L}}(f)=j(x)$. Giving the graph norm to domain $\left(\bar{\partial}_{\mathscr{L}}\right)$, we can apply the open mapping theorem to $\bar{\partial}_{\mathscr{L}}:\left\{g \in\right.$ domain $\left.\left(\bar{\partial}_{\mathscr{L}}\right): g \perp \operatorname{kernel}\left(\bar{\partial}_{\mathscr{L}}\right)\right\} \rightarrow \operatorname{range}\left(\bar{\partial}_{\mathscr{L}}\right)$ and conclude that the sequence $\left\{f_{n}\right\}$ converges to $f$ in $\mathscr{L}^{0,0}(\widetilde{X})$.

Explicit calculation of the volume form associated with $\gamma$ shows that a function is $L_{\gamma}^{2}$ if and only if it is $L^{2}$ away from $Z_{\gamma}$ and its product with the appropriate local defining functions of $Z_{\gamma}$ are $L_{\text {loc }}^{2}$ in neighborhoods of points in $Z_{\gamma}$. That is, $i: L_{\gamma}^{0,0}(\tilde{X}) \rightarrow \mathscr{L}^{0,0}(\tilde{X})$ is an isomorphism of topological vector spaces. For each $n i^{-1}\left(f_{n}\right)-F_{n}$ is in the kernel of the maximal $\bar{\partial}$-operator on $\tilde{X}-E$. It follows that for each $n i^{-1}\left(f_{n}\right)=F_{n}+c_{n}$ where $c_{n}$ is a constant function on $\widetilde{X}-E$ [10]. Because both $F_{n}$ and $c_{n}$ are in the domain of $\bar{\partial}_{c}, i^{-1}\left(f_{n}\right)$ is also. Thus we have a sequence $\left\{i^{-1}\left(f_{n}\right)\right\}$ convergent to $i^{-1}(f)$ in $L_{\gamma}^{0,0}(\tilde{X})$ and contained in domain $\left(\bar{\partial}_{c}\right)$. The sequence $\left\{\bar{\partial}_{c}\left(i^{-1}\left(f_{n}\right)\right)\right\}$ converges in $L_{\gamma}^{0,1}(\tilde{X})$ to $x$. Thus $x \in \operatorname{range}\left(\bar{\partial}_{c}\right)$.

Lemma 2.5. The cohomology of $\left\{L_{\gamma}^{0, *}(\widetilde{X}), \bar{\partial}_{c}\right\}$ at the $(0,1)$ stage is isomorphic to the cohomology of $\left\{A_{c}^{0, *}(\widetilde{X}), \bar{\partial}_{c}\right\}$ at the $(0,1)$ stage.

Proof. By showing that $\bar{\partial}_{c}^{0,0}$ has closed range, we have shown that the $(0,1)$ cohomology of $\left\{L_{\gamma}^{0, *}(\widetilde{X}), \bar{\partial}_{c}\right\}$ is represented by the intersection of kernel $\left(\bar{\partial}_{c}^{0,1}\right)$ and kernel $\left(\left(\vec{\partial}_{c}^{0,0}\right)^{*}\right)$. Elliptic regularity applied to $\bar{\partial}_{c}^{0,1}+\left(\vec{\partial}_{c}^{0,0)}\right)^{*}$ implies that $A_{c}^{0,1}(\widetilde{X})$ contains a representative of every $(0,1)$-cohomology class of $\left\{L_{\gamma}^{0, *}(\widetilde{X}), \bar{\partial}_{c}\right\}$. Then elliptic regularity applied to $\bar{\partial}_{c}^{0,0}$ shows that the preimages under $\bar{\partial}_{c}^{0,0}$ of forms smooth on $\widetilde{X}-E$ are smooth. We can conclude that the $(0,1)$-cohomology of $\left\{A_{c}^{0, *}(\tilde{X}), \bar{\partial}_{c}\right\}, H_{c}^{0,1}(\tilde{X})$, is isomorphic to the $(0,1)$-cohomology of $\left\{L_{\gamma}^{0, *}(\tilde{X}), \bar{\partial}_{c}\right\}$.

Proposition 2.6. $\left\{A_{E}^{0, *}(\tilde{X}), \bar{\partial}\right\} \rightarrow\left\{A_{c}^{0, *}(\tilde{X}), \bar{\partial}_{c}\right\}$ induces $H^{0,1}(\tilde{X}) \cong H_{c}^{0,1}(\tilde{X})$. Proof. $\left\{A_{E}^{0, *}(\tilde{X}), \bar{\partial}\right\} \rightarrow\left\{A_{c}^{0, *}(\tilde{X}), \bar{\partial}_{c}\right\} \rightarrow\left\{\right.$ domain $\left.\left(\bar{\partial}_{\mathscr{L}}^{0, *}\right), \bar{\partial}_{\mathscr{L}}\right\}$ induces $H^{0,1}(\tilde{X}) \rightarrow H_{c}^{0,1}(\widetilde{X}) \rightarrow(0,1)-\bar{\partial}_{\mathscr{L}^{-c o h o m o l o g y} \text {, and the composed map }}$

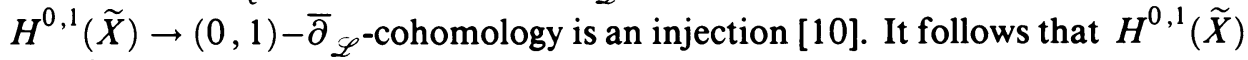
$\rightarrow H_{c}^{0,1}(\tilde{X})$ is an injection. 
Because Lemma 2.5 showed that $H_{c}^{0,1}(\widetilde{X})$ is isomorphic to the intersection of kernel $\left(\bar{\partial}_{c}^{0,1}\right)$ and kernel $\left(\left(\widetilde{\partial}_{c}^{0,0}\right)^{*}\right)$, we can use the Hodge *-operator to obtain an isomorphism of $H_{c}^{0,1}(\widetilde{X})$ with the intersection of kernel $\left(\bar{\partial}: L_{\gamma}^{2,1}(\tilde{X}) \rightarrow\right.$ $\left.L_{\gamma}^{2,2}(\widetilde{X})\right)$ and the orthogonal complement of range $\left(\bar{\partial}: L_{\gamma}^{2,0}(\widetilde{X}) \rightarrow L_{\gamma}^{2,1}(\widetilde{X})\right)$ [3]. (The $\bar{\partial}$-operators we use on $(2, *)$-forms are maximal.) Comparison of the $L_{\gamma}^{2}$ and $L^{2}$ conditions on $\tilde{X}-E$ shows that there is a commutative diagram:

$$
\begin{aligned}
& L_{\gamma}^{2,0}(\tilde{X}) \stackrel{\bar{D}}{\rightarrow} \quad L_{\gamma}^{2,1}(\tilde{X}) \stackrel{\bar{D}}{\rightarrow} \quad L_{\gamma}^{2,2}(\tilde{X})
\end{aligned}
$$

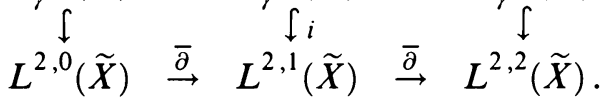

On forms of type $(2,0)$ the $L_{\gamma}^{2}$ and $L^{2}$ metrics are equivalent. Thus any $w \in L_{\gamma}^{2,1}(\tilde{X})$ satisfying $i(w) \in \operatorname{range}\left(\bar{\partial}: L^{2,0}(\tilde{X}) \rightarrow L^{2,1}(\tilde{X})\right)$ is actually in range $\left(\bar{\partial}: L_{\gamma}^{2,0}(\widetilde{X}) \rightarrow L_{\gamma}^{2,1}(\widetilde{X})\right)$. Composing $i$ with the Hodge $*$-operator, we get an injection $H_{c}^{0,1}(\widetilde{X}) \rightarrow H^{2,1}(\widetilde{X})$. Because the finite-dimensional vector spaces $H^{2,1}(\widetilde{X})$ and $H^{0,1}(\widetilde{X})$ are isomorphic, our injections $H^{0,1}(\widetilde{X}) \rightarrow$ $H_{c}^{0,1}(\widetilde{X})$ and $H_{c}^{0,1}(\widetilde{X}) \rightarrow H^{2,1}(\widetilde{X})$ prove that $H^{0,1}(\widetilde{X})$ is isomorphic to $H_{c}^{0,1}(\widetilde{X})$. This completes the proofs of Proposition 2.6 and Theorem 2.1.

\section{Curves}

In this section $X$ is a curve with smooth part $U . U$ has the metric induced from the ambient projective space. In this section we show that the cohomology groups of the minimal $\bar{\partial}$-complex of $(0, *)$-forms on $U$ are isomorphic to the corresponding groups $H^{0, *}(\tilde{X})$ on the desingularization $\tilde{X}$ of $X$. The minimal $\bar{\partial}$-complex on $U$ is the complex $\left\{A_{c}^{0, *}(U), D_{c}\right\}$. Again we work with the isomorphic complex $\left\{A_{c}^{0, *}(\widetilde{X}), \bar{\partial}_{c}\right\}$.

Theorem 3.1. Let $X$ be a curve with desingularization $\pi: \widetilde{X} \rightarrow X$. The inclusion of complexes

$$
\left\{A_{E}^{0, *}(\tilde{X}), \bar{\partial}\right\} \subset\left\{A_{c}^{0, *}(\widetilde{X}), \bar{\partial}_{c}\right\}
$$

established in Proposition 1.17 induces isomorphisms

$$
\operatorname{kernel}(\bar{\partial}) \cong \operatorname{kernel}\left(\bar{\partial}_{c}\right) \text { and } \operatorname{cokernel}(\bar{\partial}) \cong \operatorname{cokernel}\left(\bar{\partial}_{c}\right) \text {. }
$$

Proof. Because a curve has conical singularities, constant functions are in the domain of minimal exterior differentiation [2]. Thus constant functions are in kernel $\left(\bar{\partial}_{c}\right)$. To see that nothing else is in kernel $\left(\bar{\partial}_{c}^{0,0}\right)$, we use an adjoint complex, just as we ended the proof of Proposition 2.6. The Hodge *operator maps kernel $\left(\bar{\partial}_{c}^{0,0}\right)$ isomorphically to the orthogonal complement of range $\left(\bar{\partial}: L_{\gamma}^{1,0}(\tilde{X}) \rightarrow L_{\gamma}^{1,1}(\tilde{X})\right)$. There is a commutative diagram:

$$
\begin{array}{ccc}
L_{\gamma}^{1,0}(\tilde{X}) & \stackrel{\bar{\partial}}{\rightarrow} & L_{\gamma}^{1,1}(\tilde{X}) \\
\downarrow & & f_{i} \\
L^{1,0}(\tilde{X}) & \stackrel{\bar{a}}{\rightarrow} & L^{1,1}(\widetilde{X}) .
\end{array}
$$


The $L^{2}$ and $L_{\gamma}^{2}$ conditions are equivalent on $(1,0)$-forms. Thus the composition of $i$ with the Hodge *-operator gives an injection $H_{c}^{0,0}(\widetilde{X}) \rightarrow H^{1,1}(\widetilde{X})$. Because $H^{1,1}(\tilde{X}) \cong H^{0,0}(\tilde{X})$, we see that $H_{c}^{0,0}(\tilde{X}) \cong \mathbf{C}$.

The proof that there is an isomorphism of cohomology groups at the $(0,1)$ stage is much like the proof of Proposition 2.3. We have a commutative diagram:

$$
\begin{array}{ccc}
L^{0,0}(\tilde{X}) & \stackrel{\bar{\partial}}{\rightarrow} & L^{0,1}(\widetilde{X}) \\
\downarrow & & \| 2 \\
L_{\gamma}^{0,0}(\widetilde{X}) & \stackrel{\bar{\partial}_{c}}{\rightarrow} & L_{\gamma}^{0,1}(\widetilde{X}) .
\end{array}
$$

This shows that $H^{0,1}(\tilde{X})$ surjects onto cokernel $\left(\bar{\partial}_{c}^{0,0}\right)$ and that range $\left(\bar{\partial}_{c}^{0,0}\right)$ is closed. We can use the Hodge *-operator to identify cokernel $\left(\bar{\partial}_{c}^{0,0}\right)$ with $\operatorname{kernel}\left(\bar{\partial}: L_{\gamma}^{1,0}(\tilde{X}) \rightarrow L_{\gamma}^{1,1}(\tilde{X})\right)$. (Elliptic regularity and the observation that the Hodge *-operator preserves smoothness show that the cokernels of $\bar{\partial}_{c}$ : $L_{\gamma}^{0,0}(\tilde{X}) \rightarrow L_{\gamma}^{0,1}(\tilde{X})$ and $\bar{\partial}_{c}: A_{c}^{0,0}(\tilde{X}) \rightarrow A_{c}^{0,1}(\tilde{X})$ are isomorphic.) We have a commutative diagram:

$$
\begin{array}{ccc}
L_{\gamma}^{1,0}(\tilde{X}) & \stackrel{\bar{\partial}}{\rightarrow} & L_{\gamma}^{1,1}(\tilde{X}) \\
\downarrow & & \downarrow \\
L^{1,0}(\tilde{X}) & \stackrel{\bar{\partial}}{\rightarrow} & L^{1,1}(\tilde{X}) .
\end{array}
$$

Because the $L^{2}$ and $L_{\gamma}^{2}$ metrics are equivalent on $(1,0)$-forms, the kernels of the $\bar{\partial}$-operators in the above diagram are isomorphic. It follows that $H_{c}^{0,1}(\tilde{X})$ is isomorphic to $H^{1,0}(\tilde{X})$ and thus to $H^{0,1}(\tilde{X})$.

\section{REFERENCES}

1. J. Cheeger, On the Hodge theory of Riemannian pseudomanifolds, Proc. Sympos. Pure Math., Vol. 36, Amer. Math. Soc., Providence, R.I., 1980, pp. 91-146.

2. J. Cheeger, M. Goresky, and R. MacPherson, $L^{2}$-cohomology and intersection homology of singular algebraic varieties, Seminar on Differential Geometry (S. T. Yau, ed.) Princeton Univ. Press, Princeton, N.J., 1982, pp. 303-340.

3. K. Friedrichs, The identity of weak and strong extensions of differential operators, Trans. Amer. Math. Soc. 55 (1944), 132-151.

4. M. Goresky and R. MacPherson, Problems and bibliography on intersection homology, Intersection Cohomology (A. Borel et al.), Birkhauser, Boston, 1984, pp. 221-233.

5. P. Griffiths and J. Harris, Principles of algebraic geometry, Wiley, New York, 1978.

6. P. Haskell, Index theory on curves, Trans. Amer. Math. Soc. 288 (1985), 591-604.

7. W. C. Hsiang and V. Pati, $L^{2}$-cohomology of normal algebraic surfaces, I, Invent. Math. 81 (1985), 395-412.

8. R. MacPherson, Global questions in the topology of singular spaces, Proc. Internat. Congr. of Mathematicians, Polish Scientific Publishers, Warsaw, 1984, pp. 213-235.

9. M. Nagase, On the heat operators of normal singular algebraic surfaces, J. Differential Geom. 28 (1988), 37-57.

10. W. Pardon, The $L_{2}-\bar{\partial}$-cohomology of an algebraic surface, Topology (to appear).

Mathematics Department, Virginia Polytechnic Institute and State University, BLACKSBURG, VIRGINIA 24061 